\title{
The thin line between health and disease: A matter of dynamical variability
}

\author{
Xavier Bornas* and Alejandro de la Torre-Luque \\ Research Institute of Health Sciences, University of the Balearic Islands, Spain
}

One key question in Medicine and other Life sciences like Psychology concerns the definition of Health. We can roughly distinguish three historical stages, each one characterized by a different concept of Health. During several decades (in fact, centuries), health was associated with steady states and regularity. Homeostasis was a major contribution to this view of Health. Indeed, unique set points were found for many physiological and psychological systems (e.g., the body temperature should be around $36.5^{\circ} \mathrm{C}$, cut-off values of neuroticism were published, and so on). When the variability around these set points was small, it was considered some noise or measurement error, and averaging was the usual technique to reduce or remove such variability. As West pointed out, "The average value is the 'gold standard' in Medicine" [1]. Large deviations from the set points, on the other hand, were seen as symptoms of disease.

With the emergence and fast development of Chaos theory, and more generally speaking the nonlinear dynamical systems theory (NDS) in the second half of the twentieth century, the equivalence between health and steady states and regularity was challenged. Two main reasons can be highlighted. First, individuals were considered everchanging systems, and therefore they should be studied and treated as moving systems whose states were not steady but fluctuating. Hence, variability was inherent to the development of individuals (and living systems in general). Second, hidden patterns were discovered in the apparently random fluctuations of many physiological systems. Among these patterns, fractal features were found, for instance, in the beating heart and in mood fluctuations. Entropy measures demonstrated the importance of taking into account not only the variability (i.e., the dispersion of observed values around the system's set point) but the time order of the observed values. Surprisingly, decreased entropy (i.e., increased regularity) was found to be an index of disease instead of pointing at health. The homeostatic view was put into question, at least as the only view about health, disease, and the regulatory mechanisms that would get the system back to the set point when deviations occur. West [1], for instance, introduced the concept of allometric control to refer to systems that have not only one but several set points at different time scales, thus revealing the existence of a built-in long-time memory process implied in their functioning. It is worth to remember that West had introduced the concept of Fractal Physiology in 1990 [2]. Variability and irregularity were no longer viewed as unhealthy along this second stage (unless they were too large, of course).

We are now in the third stage, where health is not associated to

Copyright: (C2016 Bornas X. This is an open-access article distributed under the terms of the Creative Commons Attribution License, which permits unrestricted use, distribution, and reproduction in any medium, provided the original author and source are credited. steady states, equilibrium, and regularity, but it cannot be associated to irregularity either, as it can reveal a disease (e.g. the respiratory functioning of panic-disorder patients is highly irregular, as it is the hypothalamus-pituitary-adrenals axis hormone- releasing in depressive patients; see [3]). Yang and Tsai [4] suggested that some pathological entities are derived from an absence of variability or complexity (e.g., cardiac functioning of anxiety-disordered patients) whereas an excess of variability (or a random-like variability) would also lead to a pathological functioning of a physiological system (e.g., the bloodoxygen intake in orbitofrontal, occipital, and postcentral brain areas among schizophrenic patients). On the other hand, a special issue of the Nonlinear Dynamics, Psychology, and Life Sciences journal was recently devoted to optimum variability, and Schuldberg [5] defined it as the most desirable level of variability in the direction of growth's or movement's system over time. Thus, the degree of variability in a system must be optimal in function of how the system maximizes adaptive benefits and reduces costs.

To conclude, healthy systems are not static but dynamic, everchanging; healthy states are not steady but fluctuating; healthy systems' fluctuations seem random but they are not, they are fractal. Health is not a matter of stability or variability but a matter of being enough (optimally) variable within rather large variability limits. Disease, thus, is what one would find beyond these limits.

\section{References}

1. West BJ (2006) Complexity. In Where medicine went wrong: rediscovering the path to complexity. World Scientific 11: 241-282.

2. West BJ (1990) Fractal physiology and chaos in medicine. Singapore: World Scientific.

3. De la Torre-Luque A, Bornas X, Balle M, Fiol-Veny A (2016) Complexity and nonlinear biomarkers in emotional disorders: a meta-analytic study. Neuroscience and Biobehavioral Reviews 68: 410-422.

4. Yang AC, Tsai SJ (2013) Is mental illness complex? From behavior to brain. Progress in Neuro-Psychopharmacology \& Biological Psychiatry 45: 253-257.

5. Schuldberg D (2015) What is optimum variability? Nonlinear Dynamics, Psychology, and Life Sciences 19: 553-568.

Correspondence to: Xavier Bornas, Research Institute of Health Sciences, University of the Balearic Islands, Spain, E-mail: xavier.bornas@gmail.com

Key words: health, homeostasis, chaos, optimum variability

Received: July 03, 2016; Accepted: July 28, 2016; Published: July 30, 2016 